\title{
A Review of the Website TeamScience.net
}

\author{
David M. Aronoff, MD and Barbara A. Bartkowiak, MLIS, MST
}

\begin{abstract}
As modern research methods have become more specialized and the true complexity of today's most pressing health issues and diseases is revealed, collaborations among scientists trained in different fields have become essential for exploring and tackling these problems. This specialization of research methods has made interdependence, joint ownership, and collective responsibility between and among scientists near requirements. These features of team science may not suit everyone, but given these current trends, it is increasingly likely that most researchers will find themselves asked to participate on or lead a research team at some point in their careers.
\end{abstract}

Bennett, Gadlin, Levine-Finley ${ }^{1}$

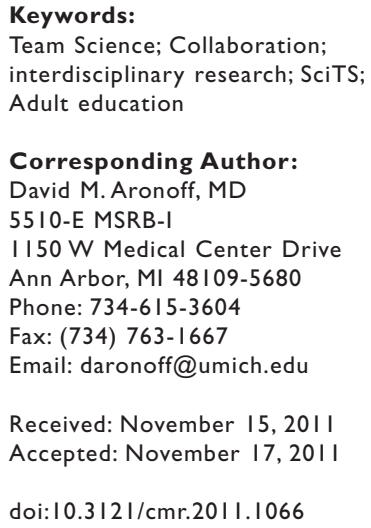

$\mathrm{T}_{\mathrm{h}}$ he process of conducting biomedical research has undergone a sea change in recentyears, with movement away from single investigator-driven "unidisciplinary" projects towards a highly collaborative, multidisciplinary or interdisciplinary structure referred to as team science. This move has been driven by many factors, motivated by the hope that "synergistic" and "holistic" studies will yield new and improved models of important biomedical problems and will translate into robust solutions. However, conducting team science presents important challenges for investigators that are sometimes more complex when compared to traditional single-investigator studies. Such challenges include plans for data sharing, communication, financial management, team leadership, and conflict resolution that can all be difficult for both established and new investigators to navigate.

In light of the growing enthusiasm for team science and an appreciation for the challenges that such an approach presents, a number of interested parties have developed tools to assist investigators in this venture. For example, the National Institutes of Health (NIH) hosts a "Collaboration and Team Science" website (https://ccrod.cancer.gov/confluence/display/NIHOMBUD/Home) with a link to their document Collaboration \& Team Science: A Field Guide. ${ }^{1}$ Moreover, a discipline referred to as the science of team science (SciTS, pronounced "sights") has emerged to define, construct, and measure interdisciplinary methods of scientific research. ${ }^{2}$

A new web-based SciTS tool is the Team Science site (http://teamscience.net/), created through NIH funding by Northwestern University. The project is called COALESCE, which stands for the CTSA Online Assistance for Leveraging the Science of Collaborative Effort. This resource represents an open suite of e-learning programs intended to facilitate learning and skill development in team science. The site is organized in a series of learning modules, message boards, and linked resources intended to enhance the user's skills at conducting and 
managing interdisciplinary (and transdisciplinary) projects. As such, it demonstrates sound adult education principles of interaction, personal experience application, and usability with good instructional design. ${ }^{3}$

The COALESCE project is strengthened by leveraging a user-friendly multimedia experience to bring together experts from different governmental and academic institutions with expertise in team science. These multimedia approaches are enhanced by nicely-produced interviews, animated movies, and text boxes. As to the layout of the site, it is a large virtual space. There is a Clinical Team Science Wing with a guided audiovisual exploration of assembling an interdisciplinary research team. This is aimed at the early career investigator. The goal of the tutorial we reviewed was to provide guidance for a young investigator attempting to secure a collaborative grant. The example provided was a team-based approach to a clinical study of exercise in patients with peripheral artery disease. Along the way this tutorial touched on critical issues for team management, including conflict resolution among investigators.

The Clinical Science Wing is laid out as a virtual building with several rooms. The rooms have titles relevant to the topics covered within them: Assembling the Team and Developing Team Leadership; Team-building Activities; Managing Conflict Upward and Downward; Sustaining Team Engagement and Communication; Evaluating Team vs. Individual Success; and a Post-Assessment room. Users click into rooms, each of which houses learning modules regarding a particular theme important to building a clinical study. Within each virtual room are icons that are selected to launch learning modules. In addition, there are occasional "Learn more" hot buttons that appear which link to even more information.

This learning exercise is helped by strong audiovisuals. The vocal tracks are clear and the visuals are aesthetically welldesigned. The use of mock collaborators, with actual names and photographs, presented an identifiable application of team science principles. As a plus, there was plenty of gender and cultural/ethnic/racial diversity. It seemed like every group photo showing investigators or research team members had an even gender split with a balance of different skin and hair colors. A nice component of this virtual tour was the judicious use of "dossiers" that could be opened via mouse clicks. The dossiers provide important contextual information and hyperlinks to outside websites (for example the NIH or the American Society of Clinical Investigation). The application of content reinforcement also appeared in feedback and assessment forms, another good instructional design component. On the whole this website is incredibly diverse and full of useful information. It is highly recommended for investigators, both junior and senior, who are interested in the evolving and emerging discipline of team science.

\section{References}

1. Bennett LM, Gadlin H, Levine-Finley S. Collaboration and Team Science: A Field Guide. National Institutes of Health: Bethesda, MD; 2010.

2. Falk-Krzesinski HJ, Contractor N, Fiore SM, Hall KL, Kane C, Keyton J, Klein JT, Spring B, Stokols D, Trochim W. Mapping a research agenda for the science of team science. Research Evaluation 2011; 20:145-158.

3. Merriam SB, Caffarella RS, Baumgartner LM. Learning in Adulthood: A Comprehensive Guide. $3^{\text {rd }}$ ed. Hoboken, NJ: Jossey-Bass; 2006.

\section{Author Affiliations}

David M. Aronoff, MD*; Barbara A. Bartkowiak, MLIS, MST'

*Division of Infectious Diseases, Department of Internal Medicine, and Program in Biomedical Sciences, University of Michigan, Ann Arbor, Michigan, USA

†eorge E. Magnin Medical Library, Marshfield Clinic, Marshfield, Wisconsin, USA 